\title{
Stretched Gelatin Phantom for Detection of Residual Dipolar Couplings in MR Spectra and Data Analysis of Carnosine
}

\author{
Karel Bernášek, ${ }^{1}$ Marián Grocký, ${ }^{1}$ Martin Burian, ${ }^{2}$ and Jan Lang ${ }^{1}$ \\ ${ }^{1}$ Department of Low Temperature Physics, Faculty of Mathematics and Physics, Charles University in Prague, \\ V Holešovičkách 2, 18000 Prague, Czech Republic \\ ${ }^{2}$ MR Unit, Department of Diagnostic and Interventional Radiology, Institute for Clinical and Experimental Medicine, \\ Vídeňská 1958/9, 14021 Prague, Czech Republic
}

Correspondence should be addressed to Jan Lang; jan.lang@mff.cuni.cz

Received 4 February 2016; Accepted 6 April 2016

Academic Editor: Vincenza Crupi

Copyright (C) 2016 Karel Bernášek et al. This is an open access article distributed under the Creative Commons Attribution License, which permits unrestricted use, distribution, and reproduction in any medium, provided the original work is properly cited.

Peak splitting due to the residual dipolar coupling (RDC) represents a potentially applicable spectral parameter for diagnostic purposes. Several of the skeletal muscle metabolites were previously reported to display the RDC splitting in in vivo MR spectra. We constructed an in vitro model consisting of mechanically stretched gelatin cylinder soaked with the muscle metabolite carnosine. We describe the preparation procedure of an upscaled $50 \mathrm{~mL}$ stretched gelatin sample with carnosine that can be used as a phantom for setting-up and testing of spectroscopic measurements of RDC in a MR scanner. We also report on analysis of the RDC splittings in ${ }^{1} \mathrm{H}$ and ${ }^{13} \mathrm{C}$ high resolution MR spectra of carnosine.

\section{Introduction}

Peak splitting due to the residual dipolar coupling (RDC) is a magnetic resonance (MR) spectral parameter which is potentially applicable for diagnostic purposes. The direct dipolar coupling in a soft matter or tissue is usually averaged out to zero by the fast changes of molecular orientation. A small residual part of this interaction is recovered if the environment is anisotropic. The induced peak splitting is additional to the common $J$-coupling. Such RDC splittings have been observed especially in the ${ }^{1} \mathrm{H}$ spectra from the skeletal muscles and the peaks displaying splitting were assigned to creatine, taurine, and carnosine $[1,2]$. The major applications of RDC stem from the fact that the observed splitting is related to the alignment tensor that quantifies the ability of the anisotropic tissue to enhance certain orientation of the observed molecule with respect to the direction of the external magnetic field. Some structural or textural properties of the muscle tissue could be revealed by measurements of RDCs.

RDCs became an important tool for determination of three-dimensional structures of biological macromolecules and, more recently, of small rigid organic molecules as well. For reviews see [3-5] and references cited therein. The species of interest are placed into anisotropic medium under the in vitro conditions. There are two major methods of invoking the anisotropy in the sample. The first one is based on the use of a liquid crystalline medium (e.g., bicelles) that become oriented in the magnetic field of the nuclear magnetic resonance (NMR) spectrometer. The second method relies on inducing a mechanical strain to a polymeric gel soaked with the solution of the species of interest.

In this paper we report on preparation of a phantom useful for setting-up and testing measurements of RDC in a MR scanner. It is based on the gelatin gel soaked with carnosine (Figure 1) that is one of the muscle metabolites, for which the observations of RDC in in vivo MR spectra were previously reported $[6,7]$. As the first step, we prepared a smaller sample for a high resolution NMR spectrometer using the apparatus for a variable gel stretching described by Kummerlöwe et al. [8]. We detected the measurable two- and three-bond ${ }^{1} \mathrm{H}^{-1} \mathrm{H}$ RDC splittings and especially one-bond ${ }^{1} \mathrm{H}_{-}{ }^{13} \mathrm{C}$ RDC splittings. As the second step, we prepared an upscaled sample with the size allowing the measurements in 


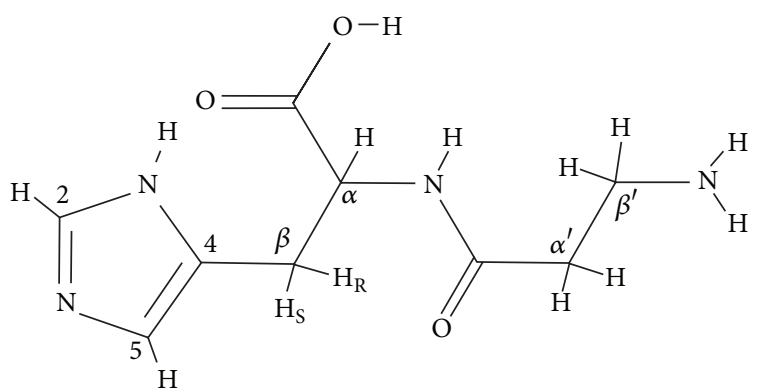

FIGURE 1: Molecular structure of carnosine with marking of carbon atoms used in this work.

a MR tomograph. We also describe analysis of the observed splittings for three samples of the mechanically stretched gelatin.

\section{Materials and Methods}

2.1. Sample Preparation. The samples were prepared using gelatin type A from Sigma-Aldrich, carnosine ( $\beta$-alanyl-Lhistidine) from Acros Organics, and deuterium oxide from Armar Chemicals. Trace amount of sodium azide from Sigma-Aldrich was added to the samples for antimicrobial protection.

The special NMR tube set for variable stretching of gel consisting of $5 \mathrm{~mm}$ outer diameter (OD) glass NMR tube open at both ends and a silicon tube $4 \mathrm{~mm}$ OD fitting into the glass tube were used for high resolution NMR measurements [8]. The nonstretched sample was about $0.15 \mathrm{~mL}$ in volume (20-25 $\mathrm{mm}$ in length, $3 \mathrm{~mm}$ in diameter).

For the high resolution NMR, three samples with gelatin concentrations of $20 \%, 30 \%$, and $40 \%$ were prepared as mixtures of raw gelatin and $0.5 \mathrm{~mol} \mathrm{dm}^{-3}$ solution of carnosine in deuterated $\mathrm{H}_{2} \mathrm{O}(90 \% \mathrm{~d})$. The initial mixture was heated up to $70^{\circ} \mathrm{C}$ until homogenous liquid was obtained. Then the mixture was cooled down in a water bath. The rigid gelatin gel was transferred into a glass funnel with the silicon tube attached. The gelatin was melted again using a hot air fan and left to descend into the silicon tube while any foam formed on top of the gelatin melt was detached. The silicon tube was closed by a PTFE plug at the bottom and a parafilm at the top and again inserted into a water bath at temperature of $50^{\circ} \mathrm{C}$ in order to get rid of any remaining bubbles. Avoiding the bubbles in the sample greatly improves the magnetic field homogeneity in the NMR measurements. The samples in the water bath were left to cool down to the room temperature. The anisotropy in the samples was invoked by stretching the silicon tube inside of the NMR tube to $120 \%, 140 \%$, and $130 \%$ of the original length for $20 \%, 30 \%$, and $40 \%$ gelatin gel samples, respectively. A small amount of the carnosine solution was added on the top of the gels in order to minimize their changes due to water evaporation in the long term.

The preparation technique was further developed in order to produce much larger phantom possible to be used in a MR tomograph. About $55 \mathrm{~mm}$ long cylinder with $30.5 \mathrm{~mm}$ inner diameter of $30 \%$ gelatin soaked with $0.2 \mathrm{~mol} \mathrm{dm}^{-3}$ carnosine solution was prepared in a way similar to the preparation of the high resolution NMR set of samples. The homogenous gel cylinder was transferred into $50 \mathrm{~mL}$ plastic centrifuge tube with $27 \mathrm{~mm}$ inner diameter, which resulted in the final length of $130 \%$ with respect to the original size. In order to facilitate the transfer, the gelatin was placed into a thin latex hose. The custom-made plastic funnel lubricated with glycerol was used to transfer the gelatin into the centrifuge tube. The latex hose enabled a certain level of stretching of the gelatin during the insertion process in order to prevent its breaking. The need of pressing on top of the gel cylinder could be thus minimized.

2.2. Measurements. High resolution NMR spectra were measured on the Bruker Avance spectrometer operating at $11.7 \mathrm{~T}$ $\left(500 \mathrm{MHz}\right.$ for ${ }^{1} \mathrm{H}, 125 \mathrm{MHz}$ for ${ }^{13} \mathrm{C}$, and $77 \mathrm{MHz}$ for ${ }^{2} \mathrm{H}$ ) using $5 \mathrm{~mm}$ TBI probe head. We acquired spectra of the anisotropic gelatin samples and a spectrum of a free solution as a reference. Ordinary one-pulse spectra were acquired for determination of the two-bond and three-bond homonuclear ${ }^{1} \mathrm{H}-{ }^{1} \mathrm{H}$ RDCs and ${ }^{2} \mathrm{H}$ residual quadrupolar couplings (RQC) in deuterated water. Two-dimensional ${ }^{1} \mathrm{H}_{-}{ }^{13} \mathrm{C}$ P.E.HSQC pulse sequence [9] was used to measure the single bond ${ }^{1} \mathrm{H}$ -

${ }^{13} \mathrm{C}$ RDCs. The $90 \mathrm{deg}$ pulse lengths were $7.5 \mu \mathrm{s}, 120 \mu \mathrm{s}$, and $14.5 \mu$ s for ${ }^{1} \mathrm{H},{ }^{2} \mathrm{H}$, and ${ }^{13} \mathrm{C}$, respectively. The polarization transfer period in P.E.HSQC was set to correspond to the total coupling of $140 \mathrm{~Hz}, 1024$ data points were acquired in the indirect $\left({ }^{13} \mathrm{C}\right)$ dimension, and 8192 data points were acquired in the direct $\left({ }^{1} \mathrm{H}\right)$ dimension. The measured free induction decays (FIDs) were processed in TopSpin 1.3. Automatic baseline correction and manual phasing of the signal were applied after the Fourier transform. RDCs were measured as a difference of splittings in isotropic ( $J$-coupling) and anisotropic (total coupling $T$ ) sample conditions. The splittings were evaluated as distances of the peak maxima of the multiplet components. All the NMR measurements were run at the temperature of $298 \mathrm{~K}$.

Localized and nonlocalized spectra of the MRI phantom were acquired on Bruker Biospec 4.7/20 operating at $200 \mathrm{MHz}$ for ${ }^{1} \mathrm{H}$ and $50 \mathrm{MHz}$ for ${ }^{13} \mathrm{C}$. The 90 deg pulse lengths were $100 \mu$ s and $50 \mu$ s for ${ }^{1} \mathrm{H}$ and ${ }^{13} \mathrm{C}$, respectively. The FID signals were processed in Paravision 4.0. ${ }^{1} \mathrm{H}$ localized spectra were measured by PRESS $[10,11]$ pulse sequence with the following parameters: echo time $6.12 \mathrm{~ms}$ (1st echo period $3.78 \mathrm{~ms}$ and 2 nd echo period $2.34 \mathrm{~ms}$ ) and time of repetition $2500 \mathrm{~ms}$, 16 averages. Prior to the localization sequence an outer volume suppression (OVS) and water suppression using VAPOR scheme [12] were run with a total duration of $650 \mathrm{~ms} .{ }^{13} \mathrm{C}$ nonlocalized spectrum was acquired in 4096 scans. The time domain data were modified by removing the first 50 points in order to suppress a broad background signal from material of the measuring probe head and the sample container. The line broadening coefficient for ${ }^{13} \mathrm{C}$ signal was set to $25 \mathrm{~Hz}$.

\section{Results and Discussion}

3.1. Anisotropic Samples Preparation. Gelatin was chosen as the basic gel medium for our samples especially due to its rather simple preparation, manipulation, and favorable 


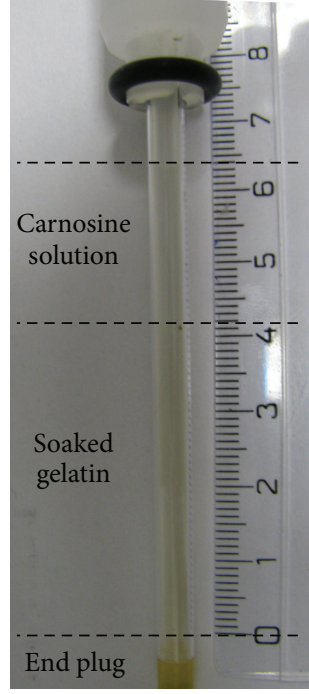

(a)

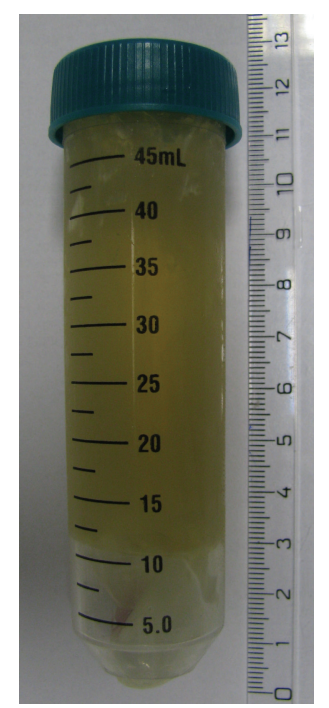

(b)

FIGURE 2: The sample for high resolution NMR: gelatin contained in the stretched silicon tube inside of the NMR tube. Dashed lines indicate interfaces boundaries of gelatin sample (a). The phantom prepared for RDC measurements in a MR tomograph (b).

mechanical properties for creating even large samples. The most important property for our purpose is reversible melting upon heating, which greatly simplifies preparation of the samples with required size and shape. It is also stiff enough thus allowing insertion of the gel cylinder into a container of a restricted diameter. A linear dependence of the measured RDCs on the stretching ratio has been reported for gelatin [13]. On the contrary, other polymeric hydrogels, such as polyacrylamide, must be irreversibly polymerized in the form of the final required unrestricted shape and subsequently pressed into the tube of restricted diameter. When a different level of compression is required, a completely new sample has to be prepared.

The spatial molecular structure of gelatin consists of the hydrogen bonded triple helices of tropocollagen, the monomer unit of the biopolymer collagen. Gelatin is a mixture of peptides and proteins produced by a partial hydrolysis of collagen extracted from skin, bones, and connective tissues. Gelatin has already been used as a tissue mimicking medium for preparation of phantoms for MR imaging and MR spectroscopy [14], and recently it has been reported as a suitable orienting medium for small molecules in the high resolution NMR spectroscopy $[13,15,16]$.

Dipeptide carnosine ( $\beta$-alanyl-L-histidine) was selected for our model samples among the other metabolites because of several factors. Its assumed prolate shape is supposed to lead to a higher degree of preferential orientation in anisotropic medium. The molecule includes rigid parts such as the imidazole ring and the peptide bond, which give better prospects for observation of RDC. Carnosine is also the biggest molecule among the small muscle metabolites, and it allows detecting the largest number of the RDC values.

3.2. The High Resolution NMR RDC Measurements. In the first stage, three samples were prepared using a special tube set $(5 \mathrm{~mm}$ OD) with the elastic silicon tube [8] suitable for the high resolution NMR spectrometer (Figure 2(a)). These small samples were utilized in order to optimize the gel preparation procedures and to enable a relatively simple detection whether the anisotropic alignment was achieved.

${ }^{1} \mathrm{H}$ high resolution NMR spectra of carnosine in the stretched gelatin (Figure 3) are well resolved and they contain all carnosine peaks in agreement with the spectrum of the carnosine water solution in isotropic conditions. There is a strong background peak centered around $0 \mathrm{ppm}$ attributed to the silicon tube that is able to overlap the solute between roughly -0.5 and $0.75 \mathrm{ppm}$. Other background peaks (except of water) are of significantly lower intensities when compared to the peaks of carnosine. The spectrum of ${ }^{1} \mathrm{H}$ spin system of carnosine was simulated in program gNMR v5.0.6.0 [17]. The chemical shifts and the $J$-coupling constants were determined from the spectrum of the free solution of carnosine. These values were plugged into the simulation of the spectra of carnosine in anisotropic conditions. In this way, the twobond and three-bond ${ }^{1} \mathrm{H}_{-}{ }^{1} \mathrm{H}$ RDCs could be determined. Due to the additive nature of $J$ and $D$, we found two theoretical options for the $D$ values for each of the threebond couplings $\mathrm{H} \beta_{\mathrm{R}}-\mathrm{H} \alpha$ and $\mathrm{H} \beta_{\mathrm{S}}-\mathrm{H} \alpha$ that provide the same spectral pattern (a total number of 4 alternatives are given in Table 1). The true solution was found by applying an assumption that signs of $D$ values should be the same for both pairs since their internuclear vectors have similar orientations. The selected solution (alternative 1) was further tested by the PALES analysis (vide infra) so that inclusion of the respective two values into the analysis brings about minimal changes of the obtained alignment tensor if compared to the same analysis employing only the set of 6 well established RDC values (i.e., excluding the values for $\mathrm{H} \beta_{R}-\mathrm{H} \alpha$ and $\mathrm{H} \beta_{S}-\mathrm{H} \alpha$ ).

We also detected seven single bond ${ }^{1} \mathrm{H}^{13}{ }^{13} \mathrm{C}$ heteronuclear RDCs (Table 1) by means of the two-dimensional 
TABLE 1: Possible combination of $\mathrm{H} \beta_{\mathrm{R}}-\mathrm{H} \alpha$ and $\mathrm{H} \beta_{\mathrm{S}}-\mathrm{H} \alpha$ residual dipolar couplings.

\begin{tabular}{lcccccc}
\hline & \multicolumn{2}{c}{$20 \%$ gelatin } & \multicolumn{2}{c}{$30 \%$ gelatin } & \multicolumn{2}{c}{$40 \%$ gelatin } \\
Alternative & $\mathrm{H} \beta_{\mathrm{R}}-\mathrm{H} \alpha$ & $\mathrm{H} \beta_{\mathrm{S}}-\mathrm{H} \alpha$ & $\mathrm{H} \beta_{\mathrm{R}}-\mathrm{H} \alpha$ & $\mathrm{H} \beta_{\mathrm{S}}-\mathrm{H} \alpha$ & $\mathrm{H} \beta_{\mathrm{R}}-\mathrm{H} \alpha$ & $\mathrm{H} \beta_{\mathrm{S}}-\mathrm{H} \alpha$ \\
& $\mathrm{RDC}(\mathrm{Hz})$ & $\mathrm{RDC}(\mathrm{Hz})$ & $\mathrm{RDC}(\mathrm{Hz})$ & $\mathrm{RDC}(\mathrm{Hz})$ & -13 & $\mathrm{RDC}(\mathrm{Hz})$ \\
\hline 1 & -10 & -8 & -14 & -11 & -13 & 4 \\
2 & -10 & 3 & -14 & 5.5 & -11 & 4 \\
3 & 3 & -8 & 5.5 & 5.5 & 4 & 4 \\
4 & 3 & 3 & 5.5 & & & 4 \\
\hline
\end{tabular}

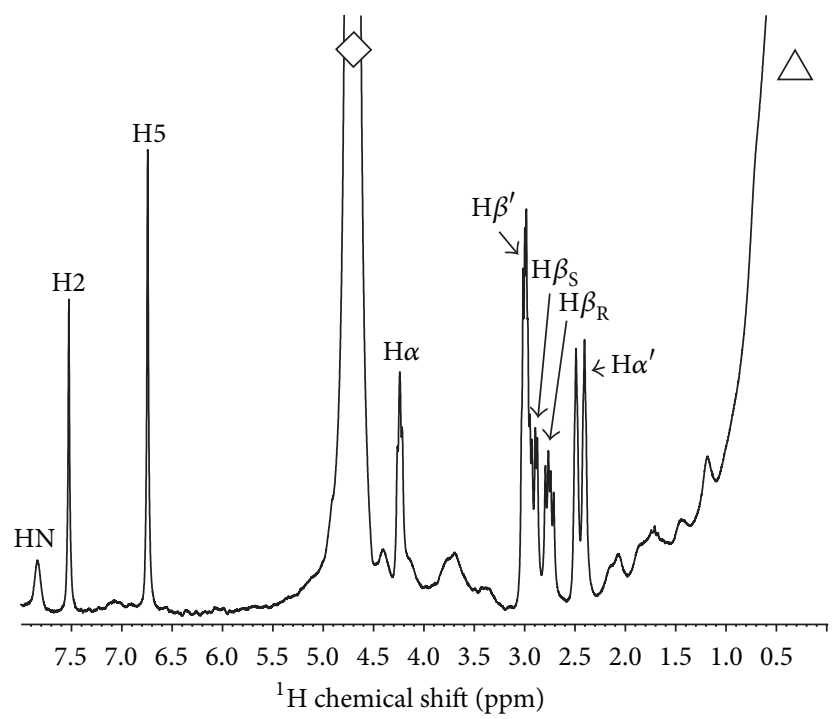

FIgURE $3:{ }^{1} \mathrm{H}$ high resolution spectrum of carnosine in $20 \%$ gelatin sample $(500 \mathrm{MHz})$. Letter-labeled peaks belong to carnosine and strong background peaks are from the silicon tube (labeled by triangle) and water (rhomb). The broad low intensity peaks are assigned to the gelatin.

P.E.HSQC [9] experiment. The RDC constant (D) for ${ }^{1} \mathrm{H}-$ ${ }^{13} \mathrm{C}$ was calculated as $D=(T-J) / 2$, where $J$ and $T$ are obtained from the measurements of isotropic and anisotropic (stretched) samples, respectively.

The data in Table 2 document that the RDC constants are of similar magnitudes for the same spin pairs in all the three different samples. The stretching ratio is the dominant factor influencing the magnitude of RDCs. The gelatin concentration increases stiffness of the gel but it has only minor effect on the size of RDCs. Supplementary Material contains the ${ }^{1} \mathrm{H}$ and ${ }^{13} \mathrm{C}$ chemical shifts in ppm (Tables S1 and S2 in Supplementary Material available online at http://dx.doi.org/10.1155/2016/4596542), the measured total couplings (Table S3), and the complete 1D and 2D spectra (Supp. Figures 1-13) of the isotropic and the stretched gelatin samples. There is no relevant systematic difference in ${ }^{1} \mathrm{H}$ or ${ }^{13} \mathrm{C}$ chemical shifts between the spectra of different samples. The error of measurement of the total coupling from the P.E.HSQC spectra is $\pm 1 \mathrm{~Hz}$ for $20 \%$ gelatin and $\pm 1.5 \mathrm{~Hz}$ for $30 \%$ and $40 \%$ gelatin due to the signal broadening caused by orienting medium and by stretching. For $1 \mathrm{D}{ }^{1} \mathrm{H}$ spectra the error is $\pm 0.5 \mathrm{~Hz}$ due to signal background and broadening by anisotropic media.

${ }^{2} \mathrm{H}$ spectra contained a single peak of the deuterated water solvent split into a doublet due to RQC of $130 \mathrm{~Hz}, 285 \mathrm{~Hz}$, and $189 \mathrm{~Hz}$ for $20 \%, 30 \%$, and $40 \%$ gelatin, respectively. The narrow line widths of the doublet components $(16 \mathrm{~Hz}$, $34 \mathrm{~Hz}$, and $25 \mathrm{~Hz}$ for $20 \%, 30 \%$, and $40 \%$ gelatin, resp.) ensure that water alignment was identical in the whole active volume of the samples. ${ }^{2} \mathrm{H}$ spectrum thus provides a simple assessment of the gel homogeneity, which is free of any spurious background.

3.3. MRS Phantom Preparation and Measurements. The MRS phantom depicted in Figure 2(b) was prepared by pressing a cylinder of $30 \%$ gelatin soaked with the carnosine solution into a $50 \mathrm{~mL}$ polypropylene centrifuge tube with the inner diameter of $27 \mathrm{~mm}$. The optimal gel parameters such as the gelatin concentration and the ratio of the stretched/unstretched gel diameters were taken from the smaller high resolution NMR samples. The size of the sample was selected so that it allows a localized measurement on $1 \mathrm{~cm}^{3}$ box.

Due to technical limitations of our MR scanner, we were able to carry out only $1 \mathrm{D}^{1} \mathrm{H}$ and ${ }^{13} \mathrm{C}$ (Figure 4) MRS measurements. Moreover, the high field part of ${ }^{1} \mathrm{H}$ spectrum suffered from a large background signals that had been caused by the utilization of the surface coil. The low field imidazole signals did not show any splitting due to ${ }^{1} \mathrm{H}-{ }^{1} \mathrm{H}$ RDC (data not shown). Following a good experience with the measurements of the one-bond heteronuclear RDCs, we acquired ${ }^{13} \mathrm{C}$ spectrum ( ${ }^{1} \mathrm{H}$ nondecoupled), and we were able to determine two values of the heteronuclear RDC unambiguously (see Table 2). Values of $\mathrm{C} \alpha-\mathrm{H} \alpha$ and $\mathrm{C} 5-\mathrm{H} 5$ are in a good agreement with the data obtained on the $30 \%$ gelatin high resolution sample. The error of measurement of total coupling in ${ }^{13} \mathrm{C}$ spectrum is estimated as $6 \mathrm{~Hz}$ due to a low signal-to-noise ratio.

3.4. Analysis of the Carnosine RDCs. We sought to check the internal consistency of the RDC values obtained from the high resolution measurements. For this purpose we attempted to calculate the RDC values based on the carnosine molecular structure by means of the program PALES (method bestFit) [18]. The method returns the alignment tensor, for which the calculated RDCs have minimal deviation from the observed ones for a given fixed molecular structure. The obtained RMSD parameter is thus a good 
TABLE 2: The measured RDC values for carnosine from the high resolution samples and from the phantom. The first 8 rows refer to histidine residue and the bottom 4 rows refer to $\beta$-alanine residue.

\begin{tabular}{|c|c|c|c|c|}
\hline Coupling pair & $\begin{array}{c}\text { RDC in } 20 \% \text { gelatin } \\
{[\mathrm{Hz}]} \\
120 \% \text { stretching ratio }\end{array}$ & $\begin{array}{c}\text { RDC in } 30 \% \text { gelatin } \\
{[\mathrm{Hz}]} \\
140 \% \text { stretching ratio }\end{array}$ & $\begin{array}{c}\text { RDC in } 40 \% \text { gelatin } \\
{[\mathrm{Hz}]} \\
130 \% \text { stretching ratio }\end{array}$ & $\begin{array}{c}\text { RDC in phantom } 30 \% \\
\text { gelatin }[\mathrm{Hz}] \\
130 \% \text { stretching ratio }\end{array}$ \\
\hline $\mathrm{C} 2-\mathrm{H} 2$ & -1.55 & -0.5 & 1 & - \\
\hline C5-H5 & 0.45 & 3 & 1.5 & 3.5 \\
\hline $\mathrm{C} \alpha-\mathrm{H} \alpha$ & 12.55 & 23.5 & 17 & 16.5 \\
\hline $\mathrm{C} \beta-\mathrm{H} \beta_{\mathrm{R}}$ & 1.3 & 9 & 4.5 & - \\
\hline $\mathrm{C} \beta-\mathrm{H} \beta_{\mathrm{S}}$ & -8.9 & -13.5 & -8.5 & - \\
\hline $\mathrm{H} \beta_{\mathrm{R}}-\mathrm{H} \beta_{\mathrm{S}}$ & -6 & -8 & -5 & - \\
\hline $\mathrm{H} \beta_{\mathrm{R}}-\mathrm{H} \alpha$ & -10 & -14 & -13 & - \\
\hline $\mathrm{H} \beta_{\mathrm{S}}-\mathrm{H} \alpha$ & -8 & -11 & -9 & - \\
\hline $\mathrm{C} \alpha^{\prime}-\mathrm{H} \alpha^{\prime}$ & 12.5 & 17.5 & 15 & - \\
\hline $\mathrm{C} \beta^{\prime}-\mathrm{H} \beta^{\prime}$ & 6.6 & 11 & 7.5 & - \\
\hline $\mathrm{H} \alpha_{\mathrm{R}}^{\prime}-\mathrm{H} \alpha_{\mathrm{S}}^{\prime}$ & 13 & 25 & 17 & - \\
\hline $\mathrm{H} \beta_{\mathrm{R}}^{\prime}-\mathrm{H} \beta_{\mathrm{S}}^{\prime}$ & 5 or $-6^{*}$ & 12 or $-14^{*}$ & 7 or $-9^{*}$ & - \\
\hline
\end{tabular}

${ }^{*}$ It was not possible to achieve unambiguous determination; both values provide identical spectral pattern.

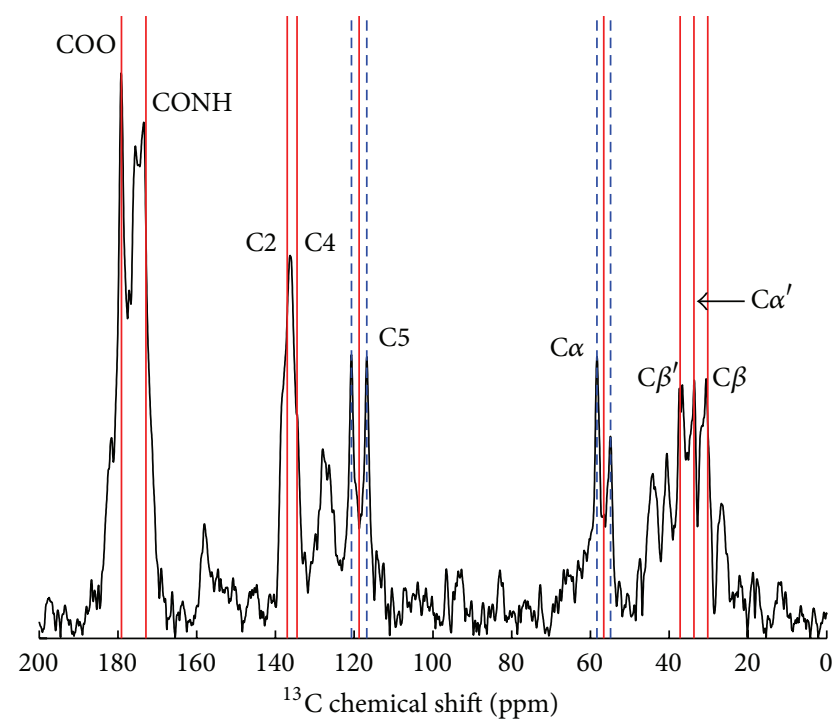

FIGURE $4:{ }^{13} \mathrm{C}$ spectrum of the gelatin phantom in magnitude mode. Solid lines indicate the position of carnosine signals in ${ }^{1} \mathrm{H}$ decoupled high resolution NMR spectrum. The measured total couplings are indicated by blue dashed lines for the peaks of $\mathrm{C} \alpha$ at $56 \mathrm{ppm}$ and $\mathrm{C} 5$ at $118 \mathrm{ppm}$.

measure of agreement between the real molecular structure in solution and an assumed theoretical one. The chemical shifts of both geminal protons of the $\beta$-alanyl $\mathrm{CH}_{2}$ groups were found nearly identical. Furthermore, we determined only a single common ${ }^{1} \mathrm{H}_{-}{ }^{13} \mathrm{C} \mathrm{RDC}$ value for each of the $\mathrm{CH}_{2}$ groups. These evidences lead us to the conclusion that the linear $\beta$-alanyl residue undergoes a fast internal dynamics which averages the observed RDC values. Therefore, we restricted our PALES analysis to the data obtained from the histidine moiety only, and the total numbers of 5 one-bond
${ }^{1} \mathrm{H}-{ }^{13} \mathrm{C}, 1$ two-bond ${ }^{1} \mathrm{H}-{ }^{1} \mathrm{H}$, and 2 three-bond ${ }^{1} \mathrm{H}-{ }^{1} \mathrm{H}$ RDCs were subject to the fit. The possible carnosine geometries were adopted from the PubChem database, CID 9369 providing 156 different stable carnosine rotamers as a result of minimization using the MMFF94s force field [19]. The complete results of the analysis are available in Table S4 in the Supporting Information. The results are evaluated according to RMSD value between the experimental and the calculated RDC values. The best obtained RMSD values are 1.5, 3.4, and 2.1 for $20 \%, 30 \%$, and $40 \%$ gelatin, respectively. Nevertheless, it is not possible to draw any strict line in order to distinguish between the structures "accepted" and those "rejected." The results must be thus considered at semiquantitative level. The employed set of the experimental value is potentially able to characterize two of the flexible torsion angles of histidine moiety-C5-C4-C $\beta-\mathrm{C} \alpha$ and $\mathrm{C} 4-\mathrm{C} \beta-\mathrm{C} \alpha-\mathrm{N}$. Among the 10 best fitting structures from each sample, there is a high population ( 8 in total) of the structures with the dihedral angles of $-147 \mathrm{deg}(\mathrm{C} 5-\mathrm{C} 4-\mathrm{C} \beta-\mathrm{C} \alpha)$ and $-78 \mathrm{deg}(\mathrm{C} 4-\mathrm{C} \beta-$ $\mathrm{C} \alpha-\mathrm{N})$. This structure corresponds well to the results of an experimental NMR study of carnosine based on J-couplings [20]. The authors found that the most populated rotamer has the G- conformation of the dihedral angle C4-C $\beta-\mathrm{C} \alpha$ $\mathrm{N}$ ( -30 to $-90 \mathrm{deg})$. Our results also support the conclusion that carnosine in solution is fairly flexible and the measured RDCs are substantially affected by motional averaging.

There is a revived interest in quantification of carnosine in muscles by means of in vivo ${ }^{1} \mathrm{H}$ MR spectroscopy [21] related also to current availability of tomographs with a high magnetic field capable of providing an excellent sensitivity. Furthermore, the peaks of imidazole protons $\mathrm{H} 2$ and $\mathrm{H} 5$ with high chemical shifts of $8 \mathrm{ppm}$ and 7 ppm, respectively, occur in an uninhabited region of spectrum since most of the resonances of the common small metabolites are found in the region $0-4.7 \mathrm{ppm}$. $\mathrm{H} 2$ is reported to be more suitable for peak integration than H5. "Orientation-dependent effects," 
that is, broadening or splitting due to RDC, were proposed as a possible reason [6]. We did not observe any RDC splitting of $\mathrm{H} 2$ or $\mathrm{H} 5$ in our high resolution NMR spectra of the stretched gelatin samples. Thus, in agreement with [21] it can be suggested that there are other relevant reasons such as shorter relaxation times with respect to $\mathrm{H} 2$, tissue compartmentation, $\mathrm{pH}$ dependence, and complexation.

When considering the muscle tissue, the stretched gelatin gel soaked with a muscle metabolite can be viewed as an oversimplified mechanical model. The alignment in gelatin is caused through interaction of carnosine with partially oriented fibers of tropocollagen. One might speculate whether the alignment characteristics of the metabolite in a skeletal muscle tissue are similar to such model. The potential of RDC for characterization of the tissue microscopic properties will be possible to exploit once ${ }^{1} \mathrm{H}^{13} \mathrm{C}$ heteronuclear experiments on the native tissue become available routinely $[22-25] .{ }^{13} \mathrm{C}$ enrichment of the metabolite or utilization of ${ }^{13} \mathrm{C}$ magnetization hyperpolarization techniques $[26,27]$ will be most probably necessary in order to increase the signal-to-noise ratio for this purpose.

\section{Conclusion}

Stretching a gelatin cylinder soaked with solution of a muscle metabolite carnosine leads to observable splittings in ${ }^{1} \mathrm{H}$ and ${ }^{13} \mathrm{C}$ MR spectra due to the residual dipolar coupling. We described preparation of the upscaled $50 \mathrm{~mL}$ stretched gelatin sample with carnosine that can be used as a phantom for setting-up and testing of the spectroscopic measurements of $\mathrm{RDC}$ in a MR scanner. We determined the set of 5 values of the two- and three-bond ${ }^{1} \mathrm{H}^{-1} \mathrm{H}$ RDCs and 7 values of one-bond ${ }^{1} \mathrm{H}^{13}{ }^{13} \mathrm{C}$ RDCs from carnosine using the methods of high resolution NMR. These values are consistent with the carnosine molecular structure experiencing a substantial level of internal flexibility although some rotamers of histidine sidechain are found to be more favored, namely, the gauche conformation of the $\mathrm{C} 4-\mathrm{C} \beta-\mathrm{C} \alpha-\mathrm{N}$ dihedral angle. There was no RDC splitting of $\mathrm{H} 2$ and $\mathrm{H} 5$ peaks observable in our ${ }^{1} \mathrm{H}$ spectra from the stretched gelatin samples. This suggests that broadening or splitting of these peaks reported for the MR spectra from muscle [6] may not be related to orientational dependence of RDC.

\section{Competing Interests}

The authors declare that they have no competing interests.

\section{Acknowledgments}

This work has been supported by the Czech Science Foundation (Project no. 13-23392S) and by the Ministry of Health, Czech Republic, Conceptual Development of Research Organization "Institute for Clinical and Experimental Medicine, IKEM, IN 00023001". The NMR spectrometer was purchased within the Project CZ.2.16/3.1.00/21566 funded by the Operational Programme Prague-Competitiveness. The authors thank Dr. Jiří Vlach for helpful suggestions regarding the preparation of orienting media, Dr. Pavel Srb for pulse sequence implementation, and Professor Burkhard Luy for generously providing them with the gel stretching apparatus.

\section{References}

[1] C. Boesch and R. Kreis, "Dipolar coupling and ordering effects observed in magnetic resonance spectra of skeletal muscle," NMR in Biomedicine, vol. 14, no. 2, pp. 140-148, 2001.

[2] C. Boesch, "Musculoskeletal spectroscopy," Journal of Magnetic Resonance Imaging, vol. 25, no. 2, pp. 321-338, 2007.

[3] M. Blackledge, "Recent progress in the study of biomolecular structure and dynamics in solution from residual dipolar couplings," Progress in Nuclear Magnetic Resonance Spectroscopy, vol. 46, no. 1, pp. 23-61, 2005.

[4] C. M. Thiele, "Residual dipolar couplings (RDCs) in organic structure determination," European Journal of Organic Chemistry, vol. 2008, no. 34, pp. 5673-5685, 2008.

[5] G. Kummerlöwe and B. Luy, "Residual dipolar couplings for the configurational and conformational analysis of organic molecules," in Annual Reports on NMR Spectroscopy, vol. 68, chapter 4, pp. 193-232, 2009.

[6] L. Schröder and P. Bachert, "Evidence for a dipolar-coupled AM system in carnosine in human calf muscle from in vivo ${ }^{1} \mathrm{H}$ NMR spectroscopy," Journal of Magnetic Resonance, vol. 164, no. 2, pp. 256-269, 2003.

[7] L. Schröder, C. Schmitz, and P. Bachert, "Molecular dynamics and information on possible sites of interaction of intramyocellular metabolites in vivo from resolved dipolar couplings in localized H-1 NMR spectra," Journal of Magnetic Resonance, vol. 171, pp. 213-224, 2004.

[8] G. Kummerlöwe, F. Halbach, B. Laufer, and B. Luy, "Precise measurement of RDCs in water and DMSO based gels using a silicone rubber tube for tunable stretching," The Open Spectroscopy Journal, vol. 2, no. 1, pp. 29-33, 2008.

[9] P. Tzvetkova, S. Simova, and B. Luy, "P.E.HSQC: a simple experiment for simultaneous and sign-sensitive measurement of $\left({ }^{1} J_{\mathrm{CH}}+D_{\mathrm{CH}}\right)$ and $\left({ }^{2} J_{\mathrm{HH}}+D_{\mathrm{HH}}\right)$ couplings," Journal of Magnetic Resonance, vol. 186, no. 2, pp. 193-200, 2007.

[10] P. A. Bottomley, "Selective volume method for performing localized NMR spectroscopy," US patent 4480228 A, 1984.

[11] P. A. Bottomley, "Spatial localization in NMR spectroscopy in vivo," Annals of the New York Academy of Sciences, vol. 508, pp. 333-348, 1987.

[12] I. Tkáč, Z. Starčuk, I.-Y. Choi, and R. Gruetter, "In vivo 1H NMR spectroscopy of rat brain at $1 \mathrm{~ms}$ echo time," Magnetic Resonance in Medicine, vol. 41, no. 4, pp. 649-656, 1999.

[13] C. Naumann, W. A. Bubb, B. E. Chapman, and P. W. Kuchel, "Tunable-alignment chiral system based on gelatin for NMR spectroscopy," Journal of the American Chemical Society, vol. 129, no. 17, pp. 5340-5341, 2007.

[14] J. R. Rice, R. H. Milbrandt, E. L. Madsen, G. R. Frank, E. J. Boote, and J. C. Blechinger, "Anthropomorphic $1 \mathrm{H}$ MRS head phantom," Medical Physics, vol. 25, no. 7, pp. 1145-1156, 1998.

[15] K. Kobzar, H. Kessler, and B. Luy, "Stretched gelatin gels as chiral alignment media for the discrimination of enantiomers by NMR spectroscopy," Angewandte Chemie-International Edition, vol. 44, no. 20, pp. 3145-3147, 2005.

[16] C. Naumann and P. W. Kuchel, " ${ }^{1} \mathrm{H}$ and ${ }^{13} \mathrm{C}$ NMR studies of glycine in anisotropic media: double-quantum transitions and 
the effects of chiral interactions," Journal of Magnetic Resonance, vol. 211, no. 1, pp. 74-79, 2011.

[17] S. Rosenfeld, "gNMR version 3.6.5," Journal of the American Chemical Society, vol. 119, no. 35, p. 8395, 1997.

[18] M. Zweckstetter, "NMR: prediction of molecular alignment from structure using the PALES software," Nature Protocols, vol. 3, no. 4, pp. 679-690, 2008.

[19] E. E. Bolton, Y. Wang, P. A. Thiessen, and S. H. Bryant, "PubChem: integrated platform of small molecules and biological activities," in Annual Reports in Computational Chemistry, R. Wheeler and D. Spellmeyer, Eds., vol. 4, chapter 12, pp. 217-241, American Chemical Society, Washington, DC, USA, 2008.

[20] J. O. Friedrich and R. E. Wasylishen, "A ${ }^{1} \mathrm{H}$ and ${ }^{13} \mathrm{C}$ nuclear magnetic resonance study of carnosine," Canadian Journal of Chemistry, vol. 64, no. 11, pp. 2132-2138, 1986.

[21] I. J. Kukurová, L. Valkovič, J. Ukropec et al., "Improved spectral resolution and high reliability of in vivo ${ }^{1} \mathrm{H}$ MRS at $7 \mathrm{~T}$ allow the characterization of the effect of acute exercise on carnosine in skeletal muscle," NMR in Biomedicine, vol. 29, no. 1, pp. 24-32, 2016.

[22] H. Watanabe, Y. Ishihara, K. Okamoto, K. Oshio, T. Kanamatsu, and Y. Tsukada, "3D localized ${ }^{1} \mathrm{H}_{-}{ }^{13} \mathrm{C}$ heteronuclear singlequantum coherence correlation spectroscopy in vivo," Magnetic Resonance in Medicine, vol. 43, no. 2, pp. 200-210, 2000.

[23] L. Mancini, G. S. Payne, and M. O. Leach, "Comparison of polarization transfer sequences for enhancement of signals in clinical ${ }^{31} \mathrm{P}$ MRS studies," Magnetic Resonance in Medicine, vol. 50, no. 3, pp. 578-588, 2003.

[24] P. C. M. Van Zijl, A. Scott Chesnick, D. Despres, C. T. W. Moonen, J. Rhiz-Cabello, and P. Van Gelderen, "In Vivo proton spectroscopy and spectroscopic imaging of $1{ }^{13} \mathrm{C}$-glucose and its metabolic products," Magnetic Resonance in Medicine, vol. 30, no. 5, pp. 544-551, 1993.

[25] T. B. Rodrigues, J. Valette, and A.-K. Bouzier-Sore, " ${ }^{13} \mathrm{C}$ NMR spectroscopy applications to brain energy metabolism," Frontiers in Neuroenergetics, vol. 5, article 9, 2013.

[26] R. A. De Graaf, D. L. Rothman, and K. L. Behar, "State of the art direct ${ }^{13} \mathrm{C}$ and indirect ${ }^{1} \mathrm{H}-\left[{ }^{13} \mathrm{C}\right]$ NMR spectroscopy in vivo. A practical guide," NMR in Biomedicine, vol. 24, pp. 958-972, 2011.

[27] J. H. Ardenkjaer-Larsen, B. Fridlund, A. Gram et al., "Increase in signal-to-noise ratio of $>10,000$ times in liquid-state NMR," Proceedings of the National Academy of Sciences of the United States of America, vol. 100, pp. 10158-10163, 2003. 

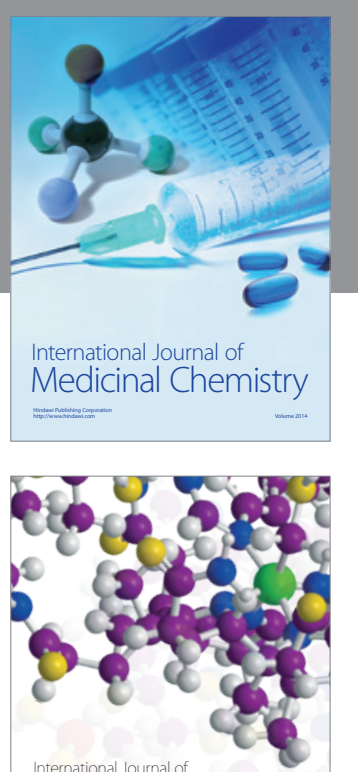

Carbohydrate Chemistry

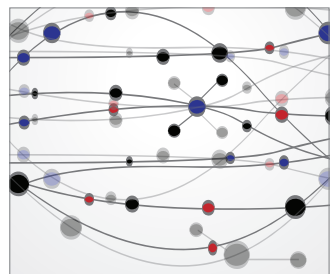

The Scientific World Journal
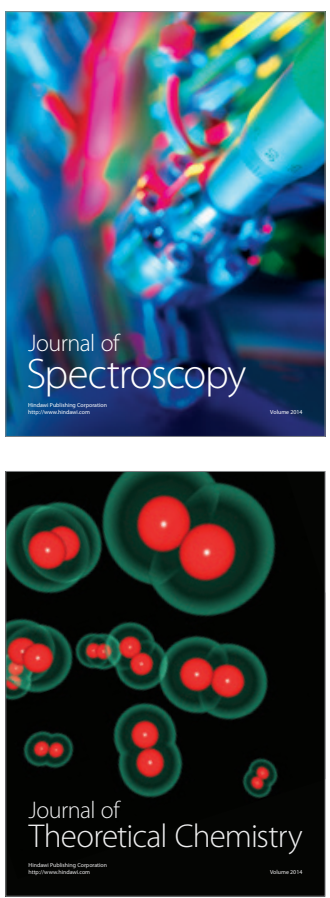
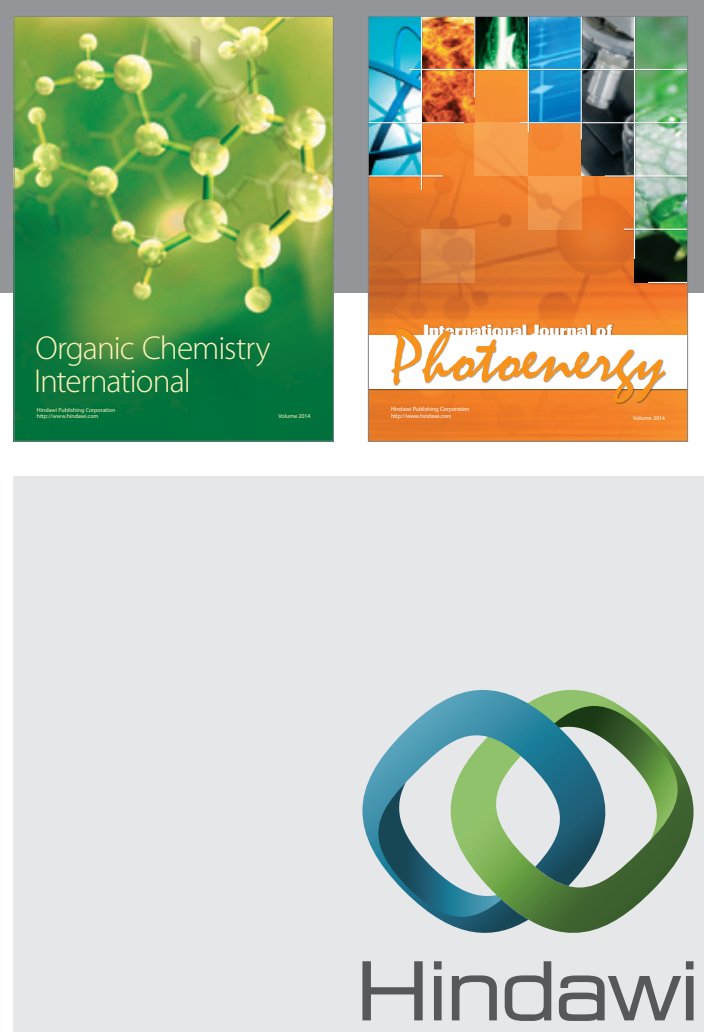

Submit your manuscripts at

http://www.hindawi.com

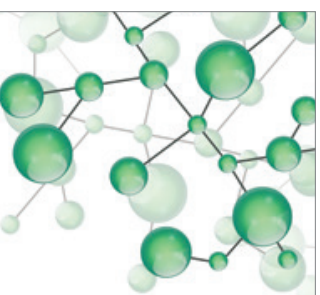

International Journal of

Inorganic Chemistry

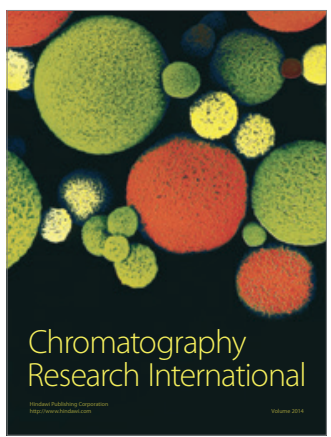

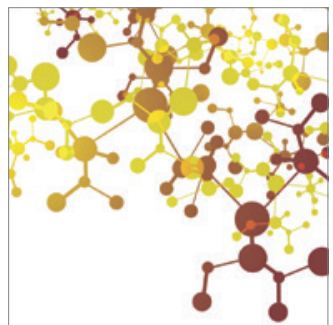

Applied Chemistry
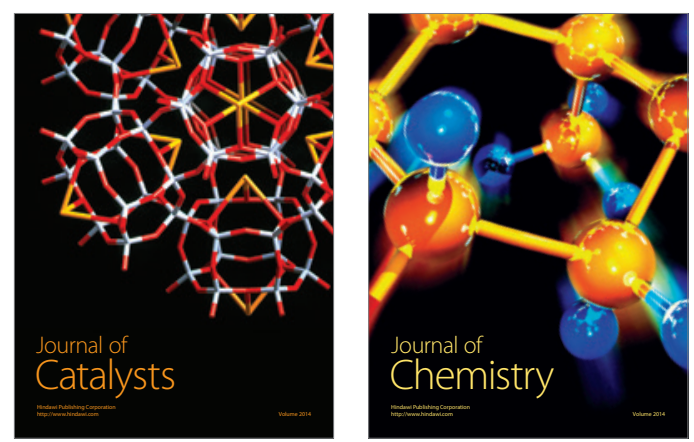
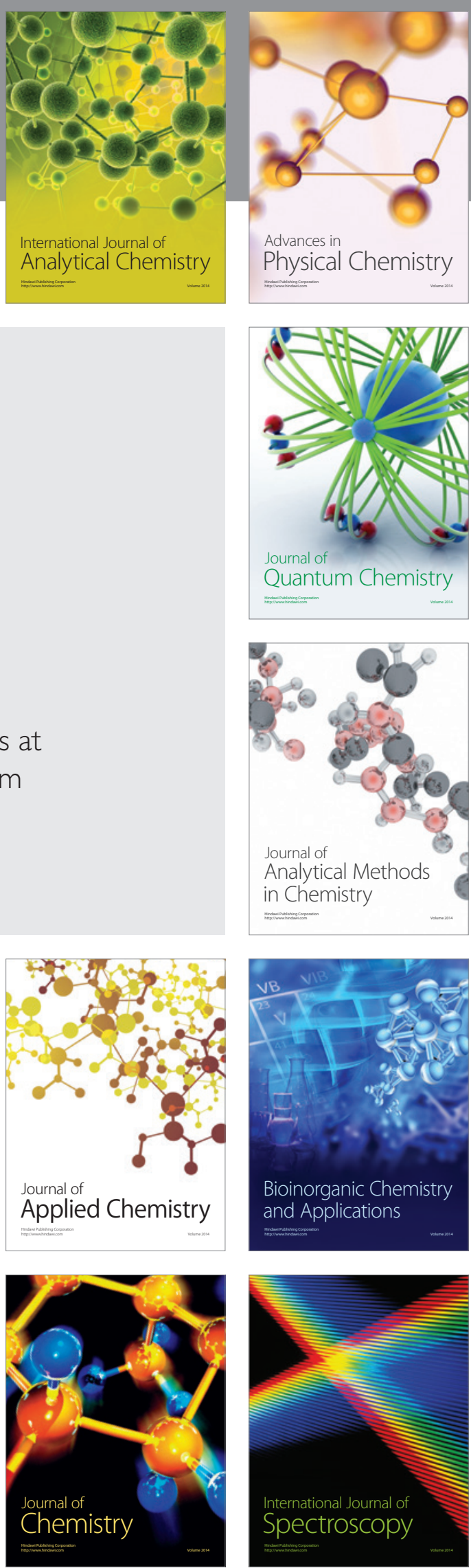